\title{
Reliability Modeling and Assessment of Component with Multiple Weak Sites under Complex Loading
}

\author{
Wenxue Qian, ${ }^{1}$ Xiaowei Yin, ${ }^{2}$ and Liyang Xie ${ }^{1}$ \\ ${ }^{1}$ School of Mechanical Engineering and Automation, Northeastern University, Shenyang 110819, China \\ ${ }^{2}$ Department of Mechanical Engineering, Shenyang Institute of Engineering, Shenyang 110136, China \\ Correspondence should be addressed to Wenxue Qian; qwx99@163.com
}

Received 21 June 2014; Revised 10 November 2014; Accepted 10 November 2014; Published 18 November 2014

Academic Editor: Leonid Shaikhet

Copyright (c) 2014 Wenxue Qian et al. This is an open access article distributed under the Creative Commons Attribution License, which permits unrestricted use, distribution, and reproduction in any medium, provided the original work is properly cited.

\begin{abstract}
A component with multiple weak sites is widely used in practical engineering and the existence of multiple weak sites can significantly decrease the component reliability. On the other hand, only a few components bear static loading and most components bear dynamic loading. In this paper, a reliability model of isomorphic component with multiple weak sites is built based on an order statistics model and the influences of strength decentrality and loading decentrality on isomorphic component with multiple weak sites are discussed. Furthermore the influence of loading times is studied in detail. The results show that unlike a component with only one weak site, not only does the failure of a component with multiple weak sites have a relationship with the safety margin, but there also exist relationships with the number of weak sites, the loading roughness, and loading times. The work in this paper is of some guiding significance in reliability design and assessment of a component with multiple weak sites under complex loading.
\end{abstract}

\section{Introduction}

Components which include multiple weak sites are widely used in practice in engineering and include compressor disks, gears, structural components of aeroplane, and turbines. Since the 1980s there have been several aircraft accidents which, following analysis of the structures of the crashed aeroplanes, have demonstrated the existence of multiple weak sites which can significantly reduce the reliability of aircraft structures [1]. Usually these components are key components in their system, and their failure will often cause disastrous results.

Because it is very important, how to predict and assess this kind of component accurately is regarded by many government regulatory bodies, designers, and scholars as essential. To this end, the European Union set up several research projects, such as the "Structural Maintenance of Ageing Aircraft, SMAAC" and "Advanced Design Concepts and Maintenance by Integrated Risk Evaluation for Aerostructures, ADMIRE," to study problems associated with multiple weak sites.

On this topic several papers [1-6] report studies on fuselage failure, mainly related to riveted lap-joints. The results showed that the residual strength of structures with multiple weak sites were decreased significantly, typically by $20 \%$, and the stress intensity factor was larger than if the impact of multiple weak sites had been ignored. A further study $[7,8]$ examined the corrosion fatigue and multiple weak sites reliability of riveted lap-jointed structures, arguing that the failure of any weak site can cause the failure of the whole structure; thus the reliability of any weak site determines the reliability of the system [9-12].

Within this field, a number of research issues are unresolved. The first is that most studies focus on riveted lapjoint structures, and these structures are relatively simple in comparison to many other components with multiple weak sites such as compressor disks and gears, more representative of the problem. The second issue is that most studies are based on deterministic methods, but in practice the sizes of weak sites, the loading, and the mechanical properties have randomness and uncertainty, and the values can be considered as random variables in accordance with certain distributions. Finally, some models are built to assess the reliability of component with multiple weak sites, but many of them do so based on the hypothesis that the failure of each 
weak site is independent. This hypothesis does not agree with the practice, as the failure of each weak site is dependent on the other weak sites [13-15].

In this paper, the modeling of component with multiple weak sites under complex loading is studied, and the failure properties of component with multiple weak sites are analyzed.

\section{Reliability Modeling and Failure Properties}

In conventional reliability assessment of a component with multiple weak sites, only one weak site is chosen to analyze the reliability and the result is considered to determine the reliability of the component. Usually this proves a too optimistic result as it has been derived by analyzing only one weak site. In reality components that contain multiple weak sites can be considered as a system, with each weak site as an element within the system. For many mechanical components, which contain more than one weak site, the failure of any one of the weak sites can cause the failure of the component. In terms of system reliability, it can be regarded as a serial system. Many practical cases show that the reliability of a component obtained through using a conventional system reliability model is markedly less than the actual reliability of the component.

The systematization method in the component reliability analysis is in accordance with the failure characteristic of a component with multiple weak sites. However, if only the conventional serial system reliability model is used to calculate the reliability of a multiple weak site component, an invalid result will be obtained. The reason for this is that the failure of each weak site is not independent but dependent. Conventional serial and parallel system reliability models derive from electronic system analysis; they are based on the hypothesis that the failure of each element is independent. For electronic systems, under certain conditions, this hypothesis can be thought to be approximately correct. However, for mechanical systems this hypothesis is almost always incorrect because the failure of an element in a system is dependent and cannot be thought of as independent from other elements in the system. Large systems such as aircraft, cars, and military tanks usually comprise of thousands of components and the relation of each to the other is serial. When using a conventional, serial system reliability model to calculate the reliability of a system, even if the reliability of each component is very high, the reliability of the system can be very low, because the system reliability is the product of the individual element reliabilities. This is not in accordance with the actual situation.

Multiple weak sites of a component usually bear the same loading, when the loading is uncertain; the failures of the weak sites are dependent on each other. The strength of each weak site and the stress imposed on each weak site are random variables and in accord with a certain distribution. Conventionally the strength of each weak site is considered as independent and identically distributed.

Suppose that the probability density function of strength is $g(s)$ and the distribution function is $G(s)$ and the probability density function of stress is $f(\delta)$ and the distribution function is $F(\delta)$. Suppose that the component comprises $n$ weak sites and the observed strength values of the weak sites are $s_{1}, s_{2}, \ldots, s_{n}$. To rank these values from smallest to biggest is as follows:

$$
s_{(1)}<s_{(2)}<s_{(3)}<\cdots s_{(n)} .
$$

From probability and statistics theory, it can be seen that $s_{(1)}, s_{(2)}, s_{(3)}, \ldots, s_{(n)}$ are the order statistics [9].

By using the multinomial distribution function, the probability density function $g_{(r)}(s)$ of the $r$ th order statistics of $n$ strength is

$g_{(r)}(s)$

$$
=\frac{n !}{(r-1) !(n-r) !}\left[\int_{-\infty}^{s} g(s) d s\right]^{r-1} g(s)\left[\int_{s}^{\infty} g(s) d s\right]^{n-r} .
$$

Here $g(s)$ is the probability density function of strength random variable $s$.

For the strengths of weak sites of a component, the most important matter is to determine if the lowest value of the strengths is less than the stress. From (2) the probability density function and the distribution function of minimal order statistics of component strength can be obtained as

$$
\begin{gathered}
g_{(1)}(s)=n g(s)\left[\int_{s}^{\infty} g(s) d s\right]^{n-1}, \\
G_{(1)}(s)=1-[1-G(s)]^{n} .
\end{gathered}
$$

When the minimum strength of all weak sites of a component is greater than the stress, then the component does not fail. Thus the reliability of the component is the probability that the minimal order statistic of component strength is greater than the stress in the domain. By using the stress-strength interference theorem, the reliability of a multiple weak site component is

$$
R=\int_{0}^{\infty} f(\delta) \int_{\delta}^{\infty} g_{(1)}(s) d s d \delta=\int_{0}^{\infty} f(\delta)[1-G(\delta)]^{n} d \delta .
$$

Model (4) is different from conventional multiple weak site component models as it considers the failure dependence of multiple weak sites bearing a common random loading.

In practical engineering, components often undertake many times effects of random loading. For those random loading, each time of effects can be considered coming from a common distribution. Usually the loading can be considered independent of each other. When a component bears many times of loading, our prime concern is the largest loading. If the largest loading is less than the lowest value of the strength, the component dose does not fail.

Suppose that the probability density function of stress is $f(\delta)$ and the distribution function is $F(\delta)$. Suppose that the loading times are $m$ and the observed stress values are $\delta_{1}, \delta_{2}, \ldots, \delta_{m}$. To rank these values from smallest to biggest is as follows:

$$
\delta_{(1)}<\delta_{(2)}<\delta_{(3)}<\cdots \delta_{(m)} .
$$


From probability and statistics theory, it can be shown that $\delta_{(1)}, \delta_{(2)}, \delta_{(3)}, \ldots, \delta_{(m)}$ are the order statistics [9].

By using the multinomial distribution function, the probability density function of $r$ th order statistics of $m$ stress is as (2).

For the stress of $n$ times action, the most important matter is to determine if the lowest value of strength is less than the stress. From (2) the probability density function and the distribution function of largest order statistics of stress can be obtained as (3):

$$
\begin{gathered}
f_{(m)}(\delta)=m f(\delta)\left[\int_{-\infty}^{\delta_{m}} f(\delta) d \delta\right]^{m-1}, \\
F_{(m)}(\delta)=[F(\delta)]^{m} .
\end{gathered}
$$

When component is undertaking multiple action of loading, if the largest order statics of loading is greater than the lowest value of the strength of the weak sites, the component will fail. So the reliability of a component with multiple weak sites is

$$
R=\int_{0}^{\infty} g_{(1)}(s) \int_{0}^{s} f_{(m)}(\delta) d \delta d s=\int_{0}^{\infty} g_{(1)}(s)[F(s)]^{m} d s .
$$

Model (7) is different from conventional multiple weak site component reliability models as it considers the failure dependence of multiple weak sites bearing the same random loading. Also it reflects the reliability changing law of the parts along with the loading times.

When using traditional stress-strength interference model to compute the reliability of a component $[16,17]$, there is a hypothesis that the reliability of a component is equal to the reliability of a weak site of a component:

$$
R=\int_{0}^{\infty} f(\delta) \int_{\delta}^{\infty} g(s) d s d \delta
$$

If we use traditional series system reliability model to calculate the reliability of a multiple weak site component, there is a hypothesis that the failures of each weak site are independent. The equation is shown as (9). In fact, the hypothesis is wrong:

$$
R=\left[\int_{0}^{\infty} f(\delta) \int_{\delta}^{\infty} g(s) d s d \delta\right]^{n}
$$

In (8) and (9), $g(y)$ is the probability density function of strength.

Model (8) does not consider the influence of the multiple weak sites and the loading times. Model (9) is based on the hypothesis that the failure of each weak site is independent. Model (7) discards the unrealistic hypothesis about independent failure and considers the dependent failure of multiple weak sites because of decentrality of common loading and the loading times.

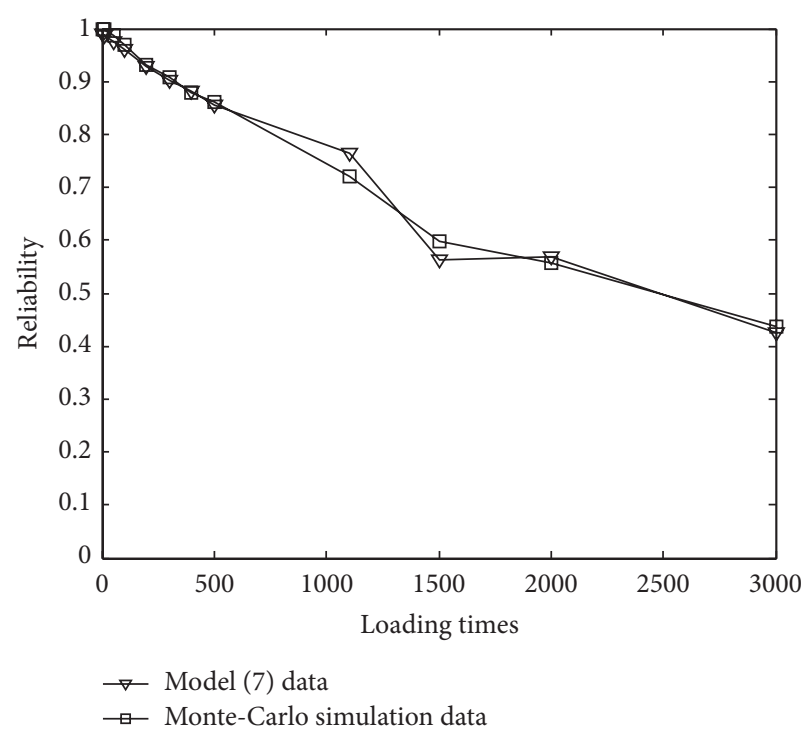

FIgURE 1: Model verifies result (sample 10000 times).

When doing reliability assessment, there are two important parameters; those are the safety margin (SM) and the loading roughness (LR) [18]; the formulas are shown as

$$
\begin{aligned}
& \mathrm{SM}=\frac{\mu_{s}-\mu_{\delta}}{\sqrt{\sigma_{\delta}^{2}+\sigma_{s}^{2}}}, \\
& \mathrm{LR}=\frac{\sigma_{\delta}}{\sqrt{\sigma_{\delta}^{2}+\sigma_{s}^{2}}},
\end{aligned}
$$

where $\mu_{s}$ is the mean strength, $\mu_{\delta}$ is the mean stress, $\sigma_{s}$ is the standard deviation of strength, and $\sigma_{\delta}$ is the standard deviation of stress.

For common reliability calculations, $\mathrm{SM}$ is also called the reliability coefficient and denoted by $\beta$. After the SM of a component is obtained, if the distributions of strength and stress are both normal distributions, then the reliability of the component can be obtained from (11). If the distribution of strength or stress does not follow normal distribution patterns, then they can be transformed into normal distributions with the equivalent normalization method:

$$
R=\Phi(\beta),
$$

where $R$ is the reliability of component and $\Phi()$ is the standard normal distribution function.

It can be seen that the traditional reliability assessment method of a component does not consider the influence of LR and the number of weak sites on reliability. For a certain component bearing different loading, if the distributions of strength and stress are both normal distributions, then as long as the SMs are the same, the reliabilities of components are equal. From model (7) it can be seen that the reliability of the component is not only related to the SM but also related to the number of weak sites and the LR of the component. 


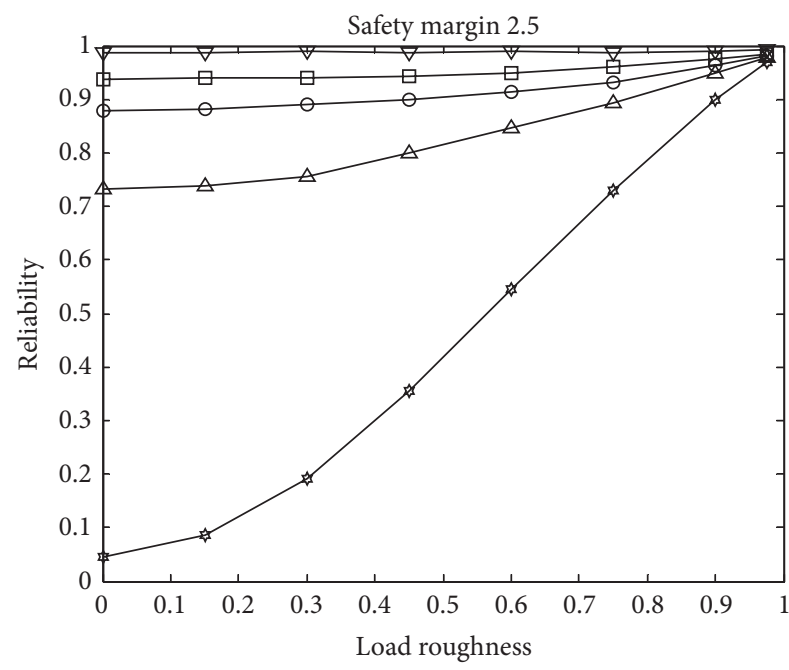

(a) 1 time

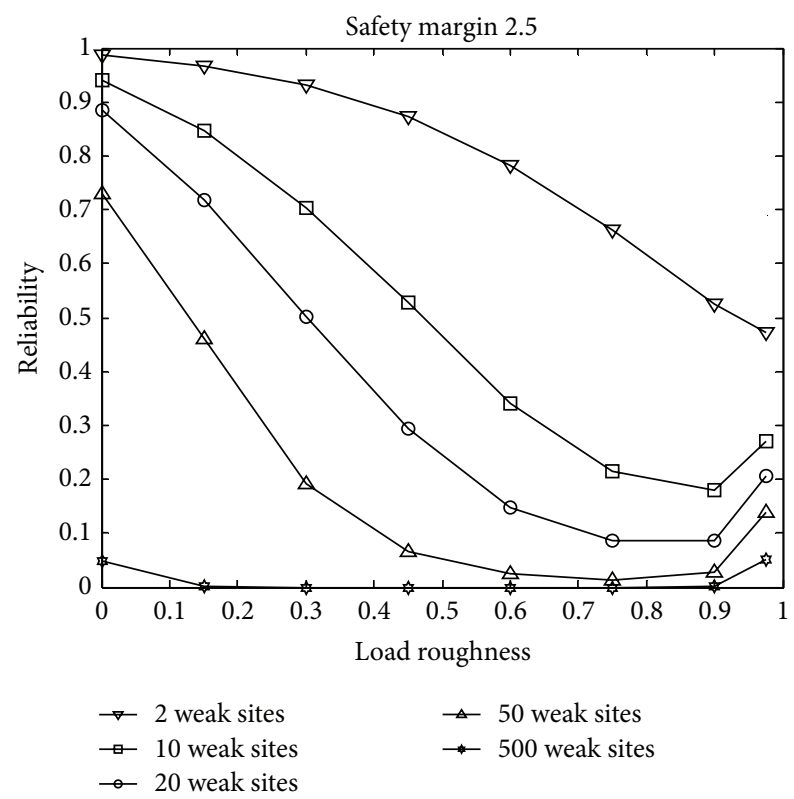

(c) 100 times

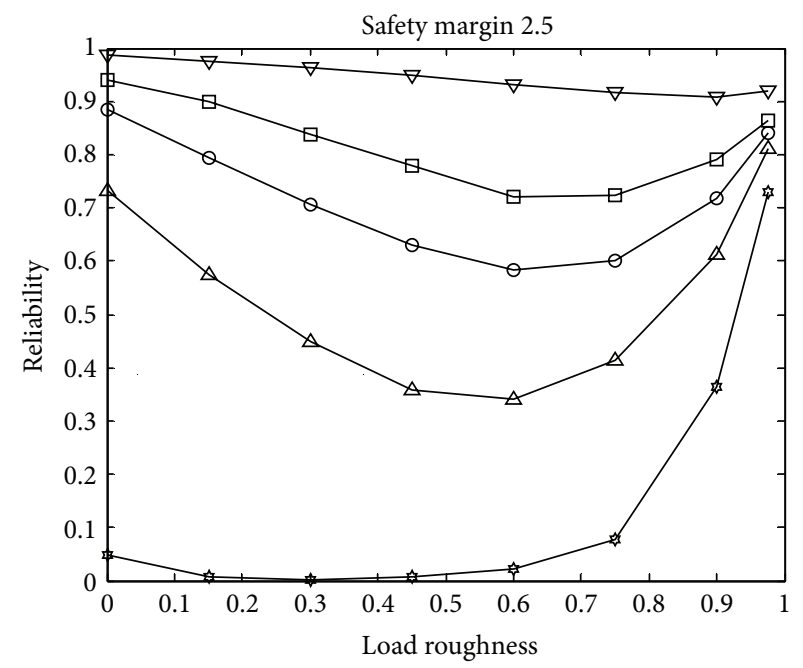

(b) 10 times

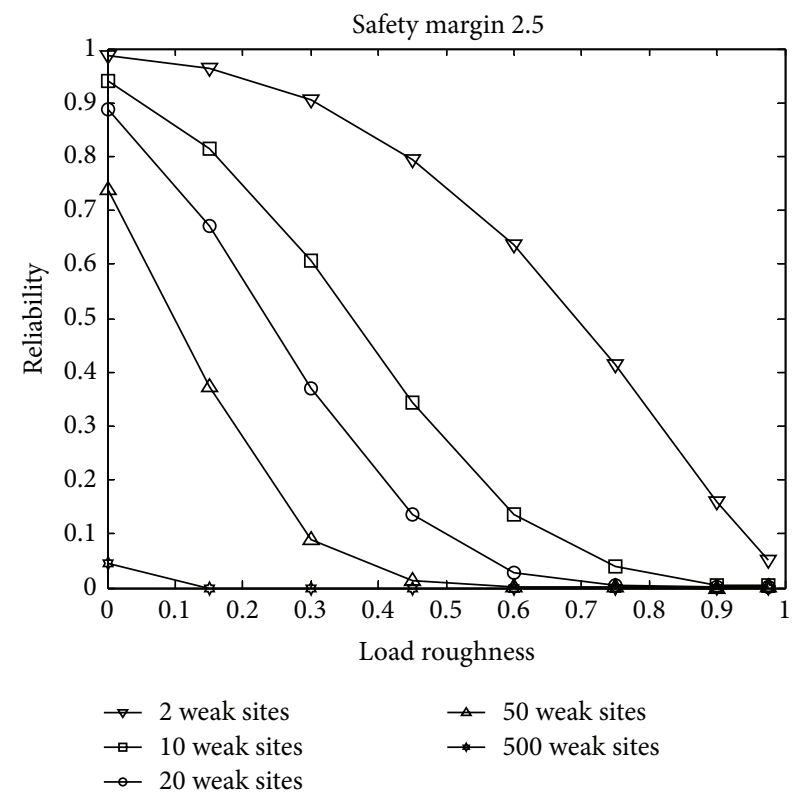

(d) 500 times

FIGURE 2: The relation between reliability and LR at SM 2.5 and different loading times.

\section{Model Validation and Parameters Analysis}

3.1. Model Validation. Here Monte Carlo method is used to verify if (7) is correct. Suppose the strength of the weak sites within a certain component is independent and follows identically distributed, normal distribution random variables. The distribution parameter of strength is $s\left(350,10^{2}\right)$, and the distribution parameter of stress is $\delta\left(200,40^{2}\right)$. When the number of weak site is $n=5$ and the loading times are from 1 to 3000, then the results from (7) and the Monte-Carlo simulation results are shown in Figure 1.

From Figure 1 it can be seen that the results of model (7) and the Monte-Carlo simulation results are consistent. This indicates the model (7) in this paper is correct.
3.2. Parameters Influence Analysis. Here the reliability of a component with multiple weak sites in relation to the number of weak sites, the LR, and loading times will be studied.

Suppose the strengths of the weak sites within a certain component are independent of each other and governed by an identically distributed normal distribution random variable. The distribution parameter of strength is $s\left(\mu_{s}, \sigma_{s}\right)$, and all the weak sites bear a common load, with the distribution parameter of stress $\delta\left(\mu_{\delta}, \sigma_{\delta}\right)$. When the number of weak sites is $n=1$, then from ( 7 ) the reliability of the component is only determined by the SM and loading times and has no relation with the LR. In order to study the influence of LR, the loading times, and the number of weak sites on the reliability of a multiple weak site component, let $\mu_{s}=360 \mathrm{MPa}$ and 


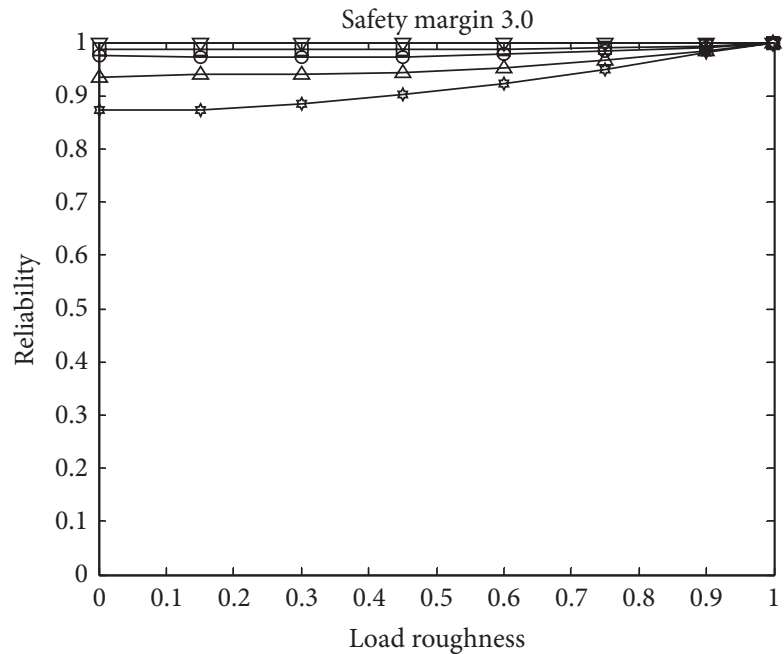

(a) 1 time

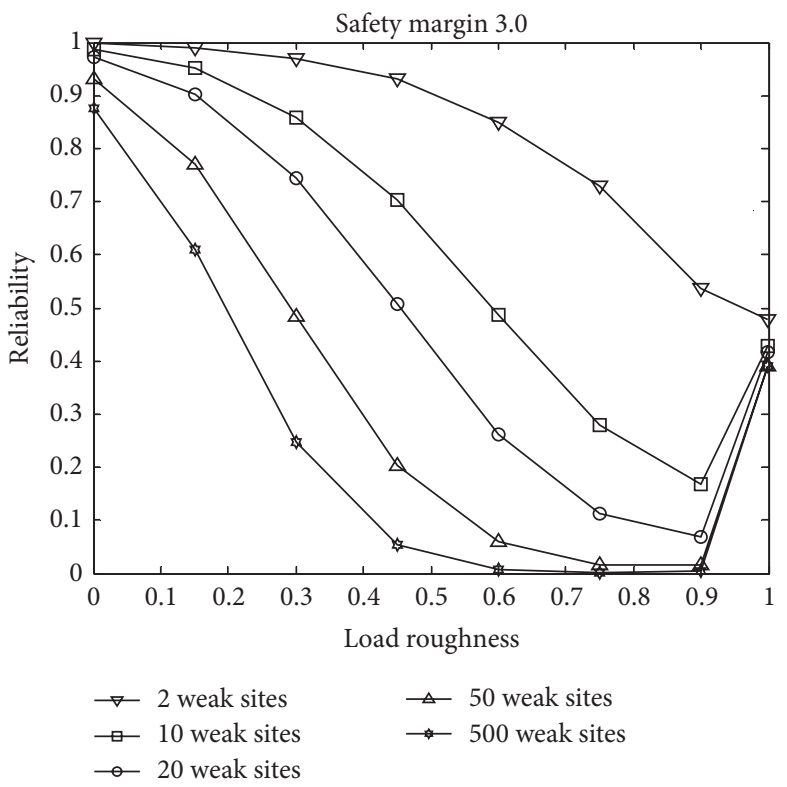

(c) 500 times

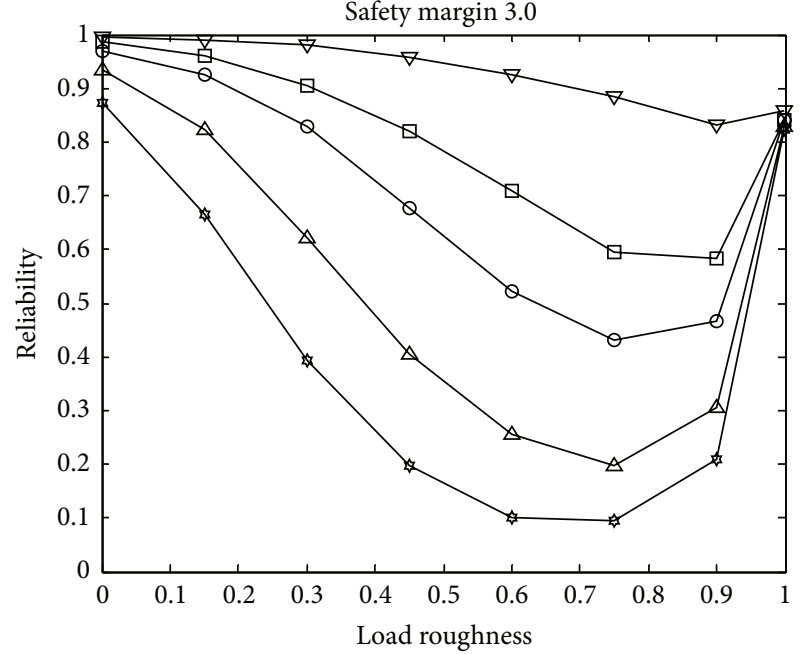

(b) 100 times

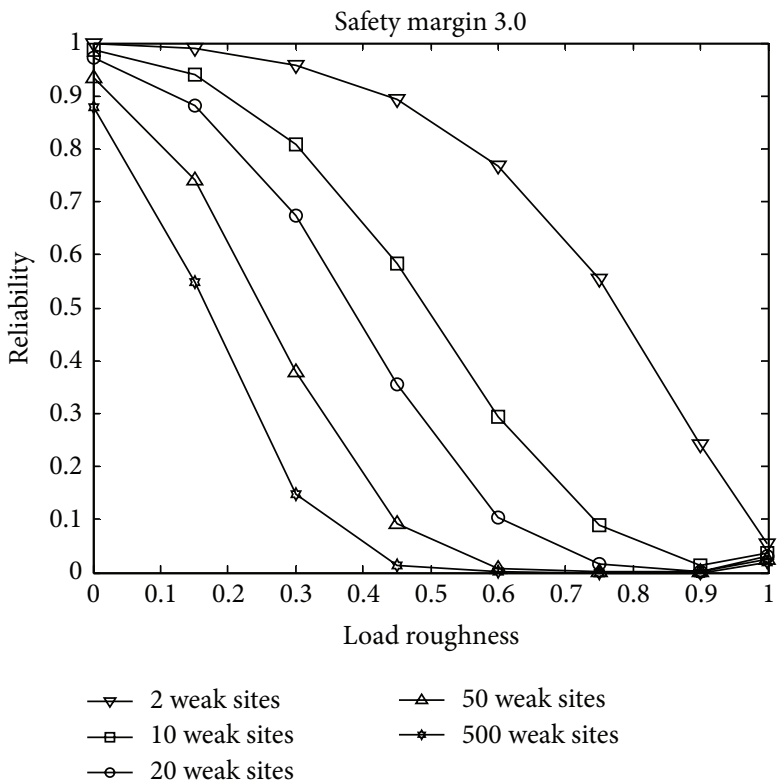

(d) 2000 times

FIGURE 3: The relation between reliability and LR at SM 3.0 and different loading times.

$\mu_{\delta}=260 \mathrm{MPa}$. When the SM of the weak site is 2.5, 3.0, 4.0 , and 5.0, then from model (7) the relationship curves of reliability, LR, and loading times can be obtained and are shown in Figures 2, 3, 4, and 5. When it is assumed that the failure of each weak site of a component is independent, then the relation curve of component reliability and the number of weak sites is shown in Figure 6.

3.3. Results Discussion. For a component with multiple weak sites, if only one weak site is considered, that is to say, the reliability of the weak site is thought of as the reliability of the component, then $R=R_{1}$, where $R$ is the reliability of the component and $R_{1}$ is the reliability of any weak site. When using a conventional serial system reliability model to calculate the reliability of a multiple weak site component, the result is $R=R_{1}^{n}$, where $n$ is the number of weak sites. However, as this result does not consider the effect of LR, the reliability is usually far less than the actual value. From Figures 2-5 it can be seen that the reliability of a component with multiple weak sites is closely related to LR, the number of weak sites within it, and loading times. To Figure 2(a), for a single load application and lower SM, such as when $\mathrm{SM}=2.5$, then when $n=50$ and $R_{1}=0.9768$, if only one weak site is considered, $R=R_{1}=0.9768$, and $R=$ $R_{1}^{n}=0.9768^{50}=0.3092$ when using a conventional serial system reliability model. In practice these two results are the upper and lower bounds of the static reliability of the component. From Figure 2(a) it can be seen that the reliability of a component increases with the increasing of LR. Further, 

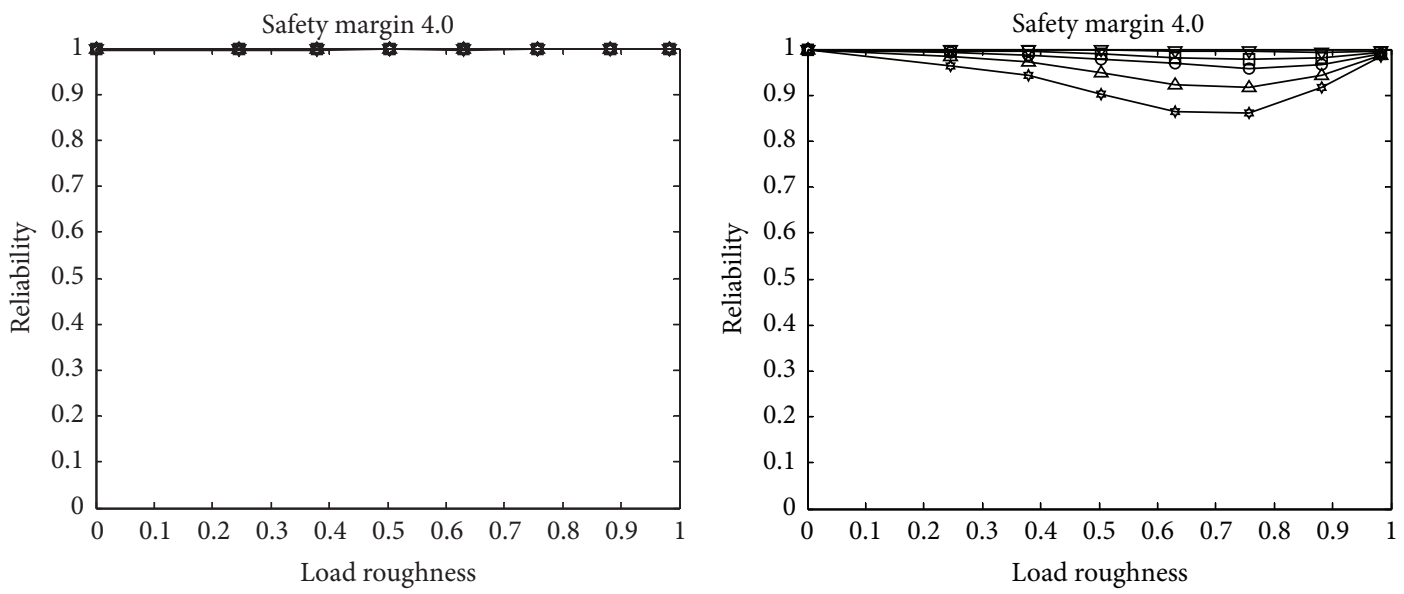

(a) 1 time

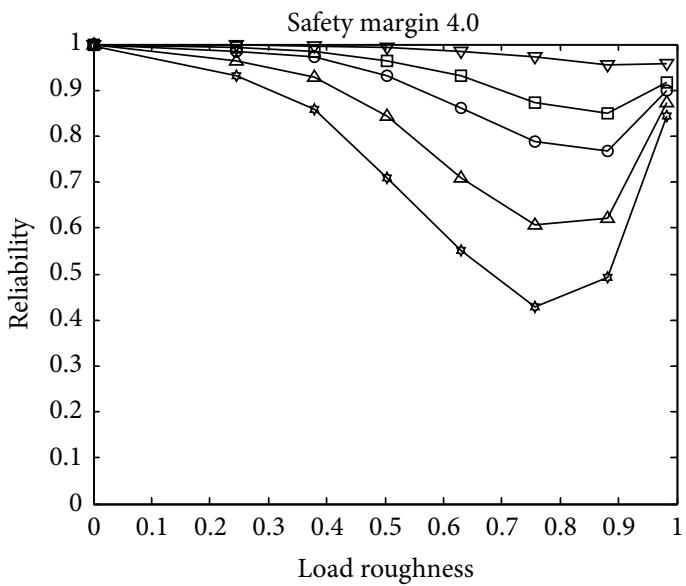

(b) 100 times

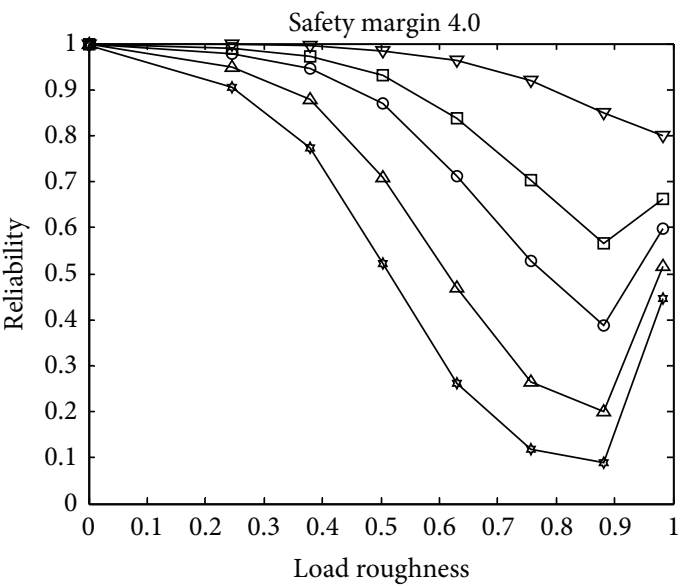

(c) 1000 times

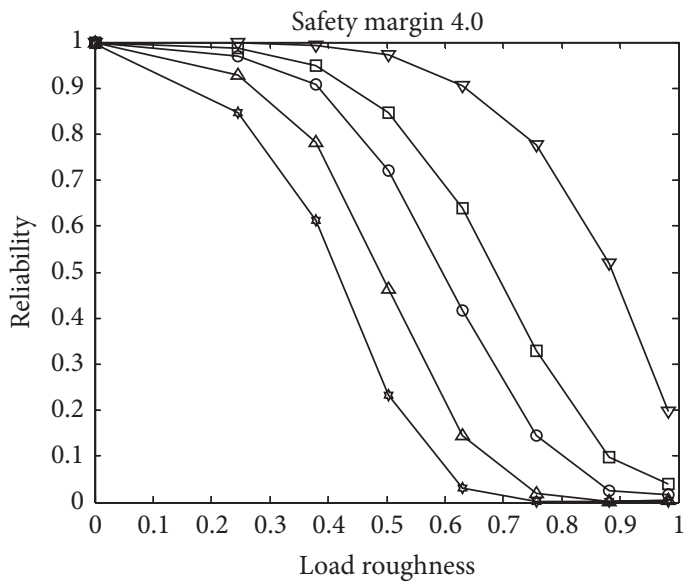

(d) 5000 times

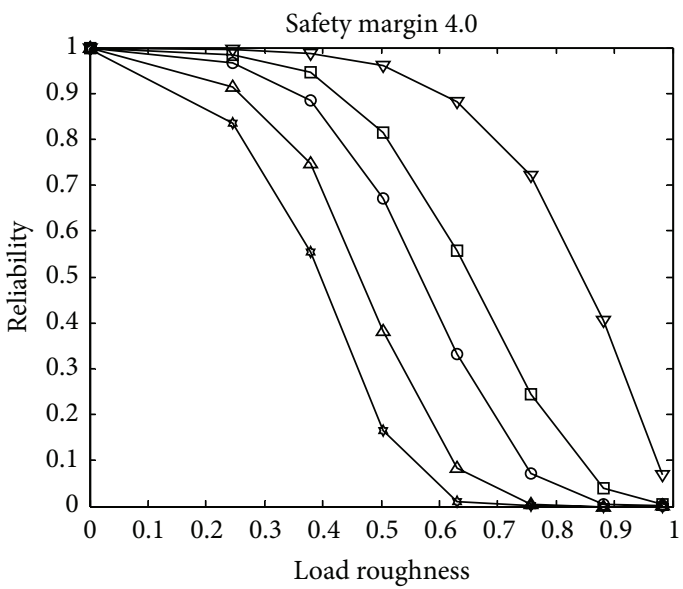

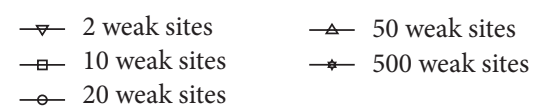

(e) 50000 times

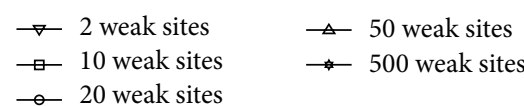

(f) 100000 times

FIGURE 4: The relation between reliability and LR at SM 4.0 and different loading times. 
Safety margin 5.0

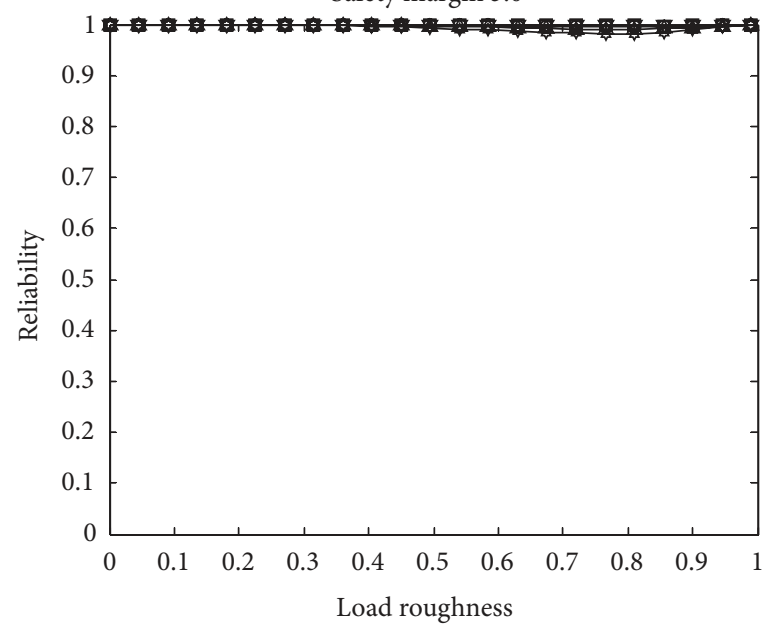

(a) 1000 times

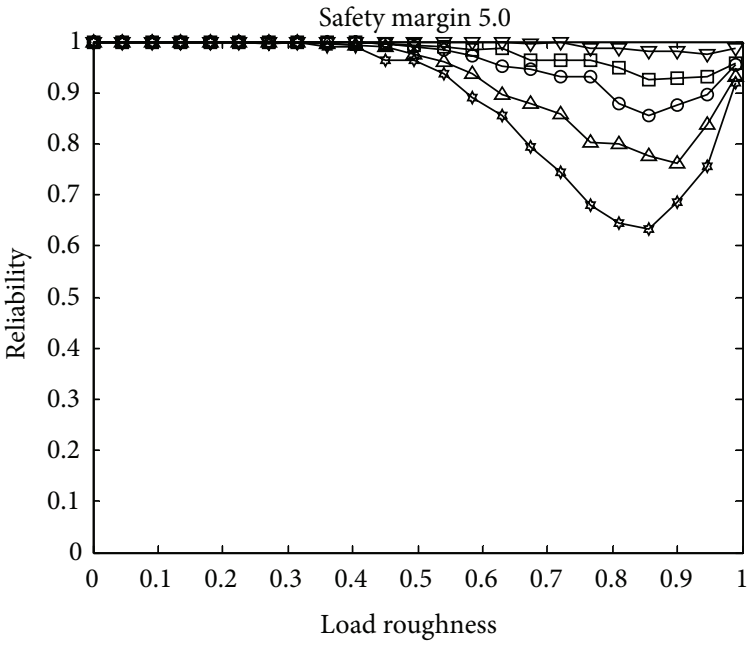

(c) 50000 times

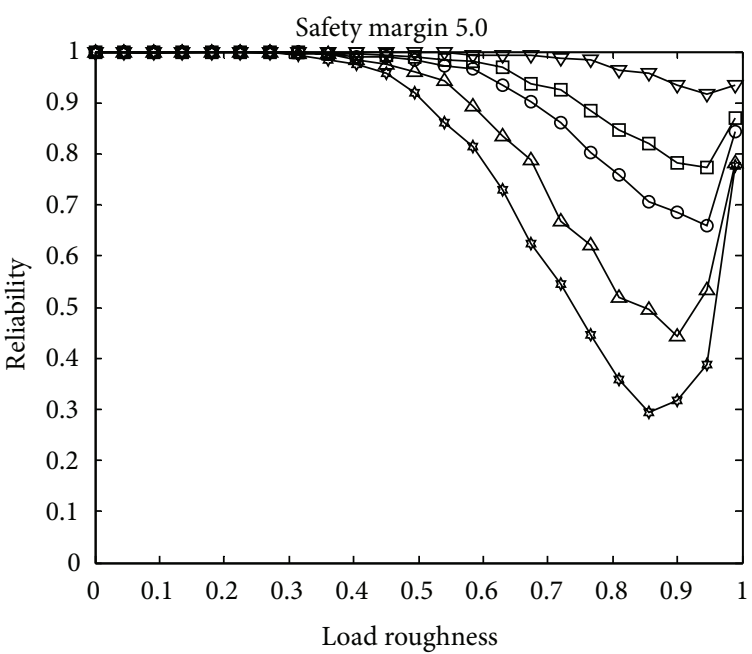

$\rightarrow 2$ weak sites

$\square-10$ weak sites

$\triangle \quad 50$ weak sites

\-500 weak sites

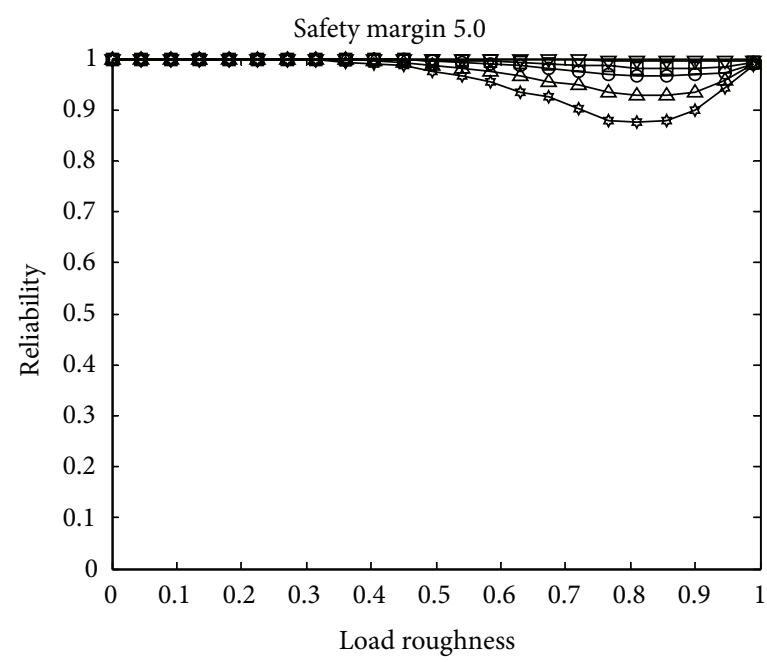

(b) 10000 times

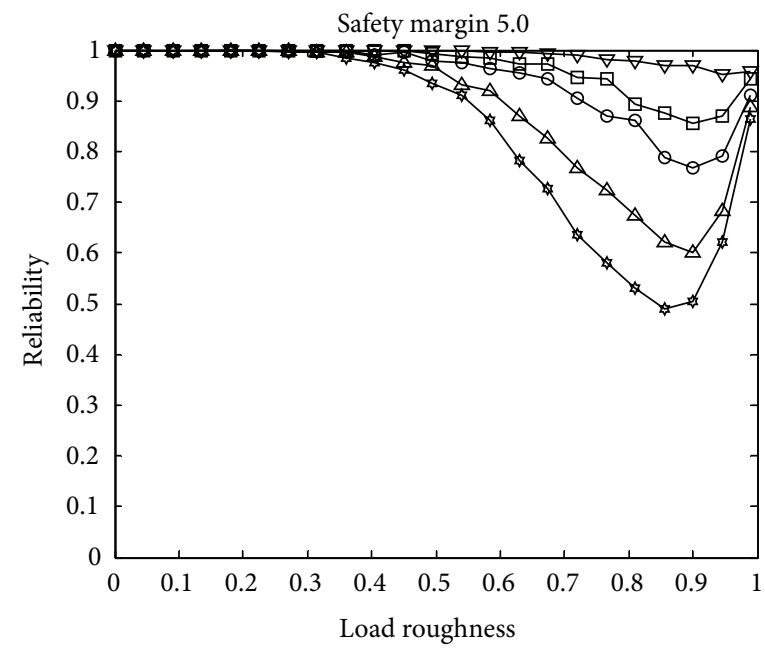

(d) 100000 times

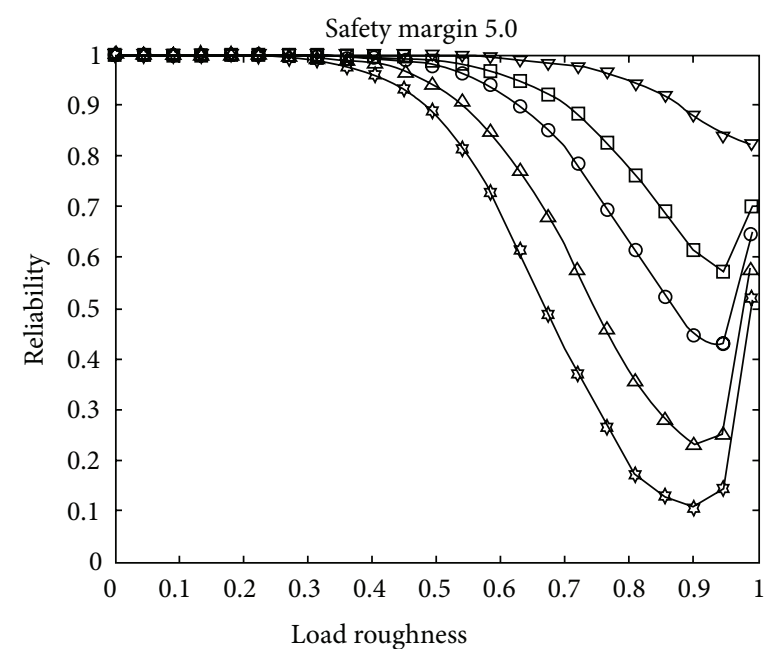

$\neg 2$ weak sites

$\square-10$ weak sites - 20 weak sites

(e) 200000 times

(f) 500000 times

Figure 5: Continued. 


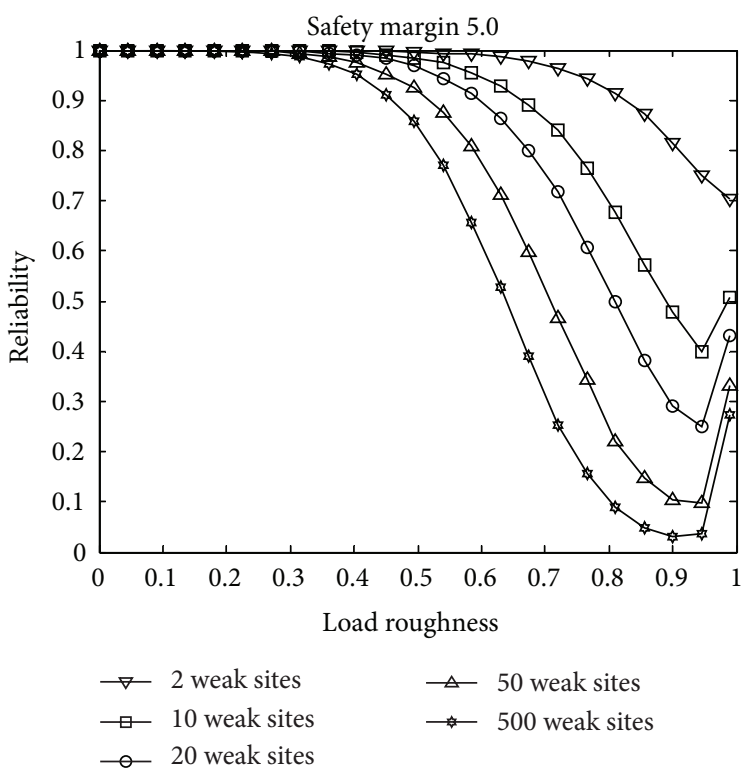

(g) 1000000 times

FIGURE 5: The relation between reliability and LR at SM 5.0 and different loading times.

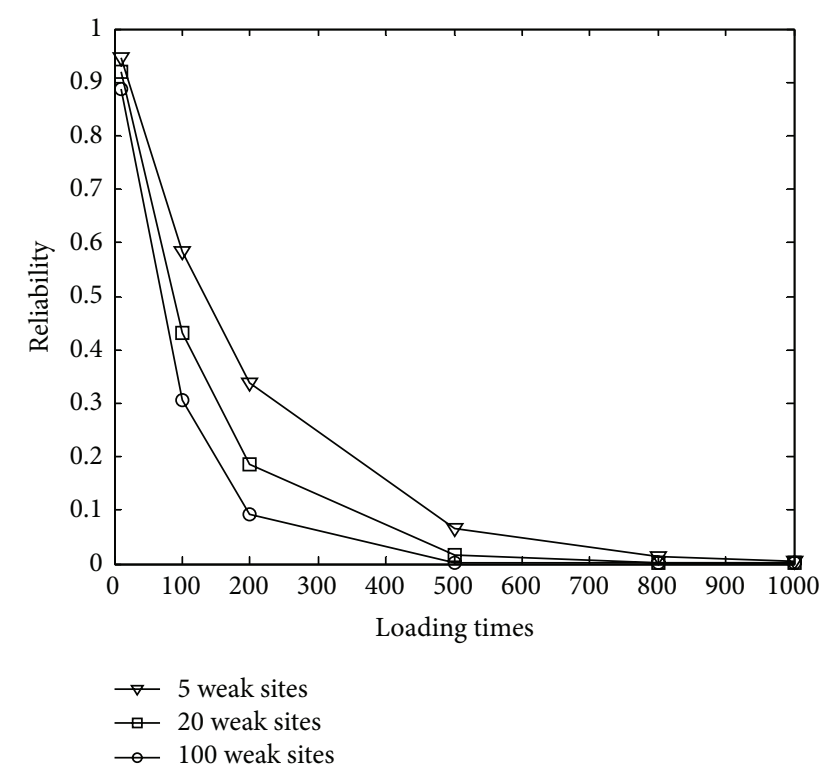

FIGURE 6: Independent failure model results.

when $\mathrm{LR}=0.3$, if $n=10, R=0.9401$, if $n=50, R=0.7573$, and when $\mathrm{LR}=0.9$, if $n=10, R=0.9743$, if $n=50, R=0.95$.

Figure 2(a) shows the relationship between reliability and LR, reliability and the number of weak sites under simple load. Figures 2(b)-2(d) and Figures 3-5 show the relationship between reliability and LR, reliability and SM, and reliability and loading times. For Figure 2(c), it can be seen that there are several characteristics: first the reliability reduces as the number of weak sites increases, second the reliability reduces with the increasing of loading times, and third the reliability decreases first and then increases as LR increases.
The static reliability of a component with multiple weak sites increases along with the increasing of LR. The reason for this is that for lower LR the failure of each weak site tends toward independence while with the increasing of LR the failure of each weak site tends toward dependence. For the reliability of a component with multiple weak sites under complex loading, the effect of LR weakened and only it can increase the reliability while the LR is greater than 0.9 as shown in Figures 5(b)-5(e).

Figure 6 shows the relationship curves of the reliability and the loading times for components under the independent failure hypothesis. It can be seen that the reliability of components decreases very quickly as a result of unreasonable assumptions with the result being too conservative and meaningless in a practical sense.

\section{Conclusion}

In this paper, a new reliability model is introduced to assess the reliability of a component with multiple weak sites under complex loading. The modeling process is without the hypothesis that the failure of each weak site is independent. Through this model it can be seen that not only the SM but also the LR and the number of weak sites have important effects on the reliability of the component with multiple weak sites. When using conventional serial system reliability models to assess the reliability of a component with multiple weak sites, the result is too conservative. Higher SM can increase the reliability of the component with multiple weak sites, and when the SM is determinate, then higher LR can decrease the sensitivity of the component reliability to the number of weak sites. For a component with multiple weak sites under complex loading, high SM is necessary and 
the effect of LR weakened. So high LR $(>0.9)$ is necessary for practical engineering components.

\section{Conflict of Interests}

The authors declare that there is no conflict of interests regarding the publication of this paper.

\section{Acknowledgments}

This work was partially supported by the National Natural Science Foundation of China (Grant nos. 51005044, 51305275, 51335003, and 51275221) and the Fundamental Research Funds for the Central Universities (Grant no. N120403014).

\section{References}

[1] H. Razia, B. Sergeevb, S. Shkarayevb, and E. Madencib, "Analysis of sandwich panels with multiple-site damage," Engineering Fracture Mechanics, vol. 64, no. 2, pp. 255-268, 1999.

[2] R. Jones, L. Molent, and S. Pitt, "Study of multi-site damage of fuselage lap joints," Theoretical and Applied Fracture Mechanics, vol. 32, no. 2, pp. 81-100, 1999.

[3] R. M. V. Pidaparti, M. J. Palakal, and Z. A. Rahman, "Simulation of structural integrity predictions for panels with multiple site damage," Advances in Engineering Software, vol. 31, no. 2, pp. 127-135, 2000.

[4] L. F. M. Silva, J. P. M. Gonçalves, F. M. F. Oliveira, and P. M. S. T. De Castro, "Multiple-site damage in riveted lapjoints: experimental simulation and finite element prediction," International Journal of Fatigue, vol. 22, no. 4, pp. 319-338, 2000.

[5] M. C. Cherry, S. Mall, B. Heinimann, and A. F. Grandt Jr., "Residual strength of unstiffened aluminum panels with multiple site damage," Engineering Fracture Mechanics, vol. 57, no. 6, pp. 701-713, 1997.

[6] P. Shi and S. Mahadevan, "Corrosion fatigue and multiple site damage reliability analysis," International Journal of Fatigue, vol. 25, no. 6, pp. 457-469, 2003.

[7] T. Zhang and M. Horigome, "Availability and reliability of system with dependent components and time-varying failure and repair rates," IEEE Transactions on Reliability, vol. 50, no. 2, pp. 151-158, 2001.

[8] J. Varghese and A. Dasgupta, "An experimental approach to characterize rate-dependent failure envelopes and failure site transitions in surface mount assemblies," Microelectronics Reliability, vol. 47, no. 7, pp. 1095-1102, 2007.

[9] L. Xie, J. Zhou, and C. Hao, "System-level load-strength interference based reliability modeling of k-out-of-n system," Reliability Engineering and System Safety, vol. 84, no. 3, pp. 311-317, 2004.

[10] W. Qian, X. Yin, and L. Xie, "System reliability allocation based on Bayesian network," Applied Mathematics and Information Sciences, vol. 6, no. 3, pp. 681-687, 2012.

[11] L. Xie, J. Zhou, and X. Wang, "Data mapping and the prediction of common cause failure probability," IEEE Transactions on Reliability, vol. 54, no. 2, pp. 291-296, 2005.

[12] J. Zhou and L. Xie, "Generating function approach to reliability analysis of structural systems," Science in China, Series E: Technological Sciences, vol. 52, no. 10, pp. 2849-2858, 2009.

[13] L. Xie, J. Liu, N. Wu, and W. Qian, "Backwards statistical inference method for P-S-N curve fitting with small-sample experiment data, International Journal of Fatigue, vol. 63, pp. 62-67, 2014.

[14] W. Zheng and X. Liyang, "Dynamic reliability model of components under random load," IEEE Transactions on Reliability, vol. 57, no. 3, pp. 474-479, 2008.

[15] P. Gao, S. Yan, L. Xie, and J. Wu, "Dynamic reliability analysis of mechanical components based on equivalent strength degradation paths," Strojniski Vestnik/Journal of Mechanical Engineering, vol. 59, no. 6, pp. 387-399, 2013.

[16] A. M. Freudenthal, M. Carrelts, and M. Shinozuka, "The analysis of structural safety," ASCE Journal of Structure Division, vol. 92, pp. 267-325, 1966.

[17] J. D. Booker, M. Raines, and K. G. Swift, Designing Capable and Reliable Products, Butterworth-Heinemann, Oxford, UK, 2001.

[18] A. D. S. Carter, Mechanical Reliability and Design, John Wiley \& Sons, Hoboken, NJ, USA, 1997. 


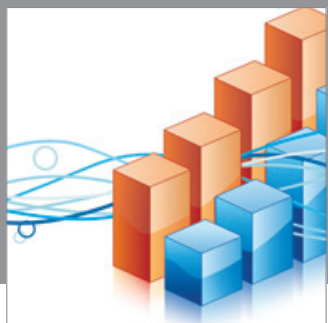

Advances in

Operations Research

mansans

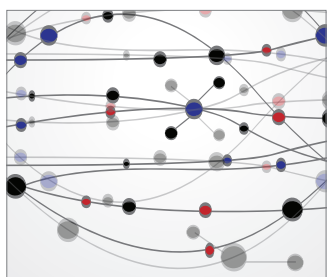

The Scientific World Journal
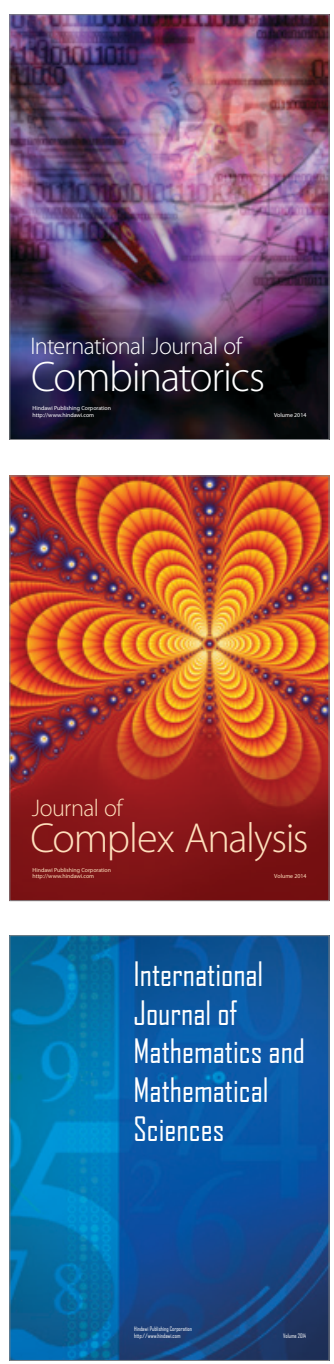
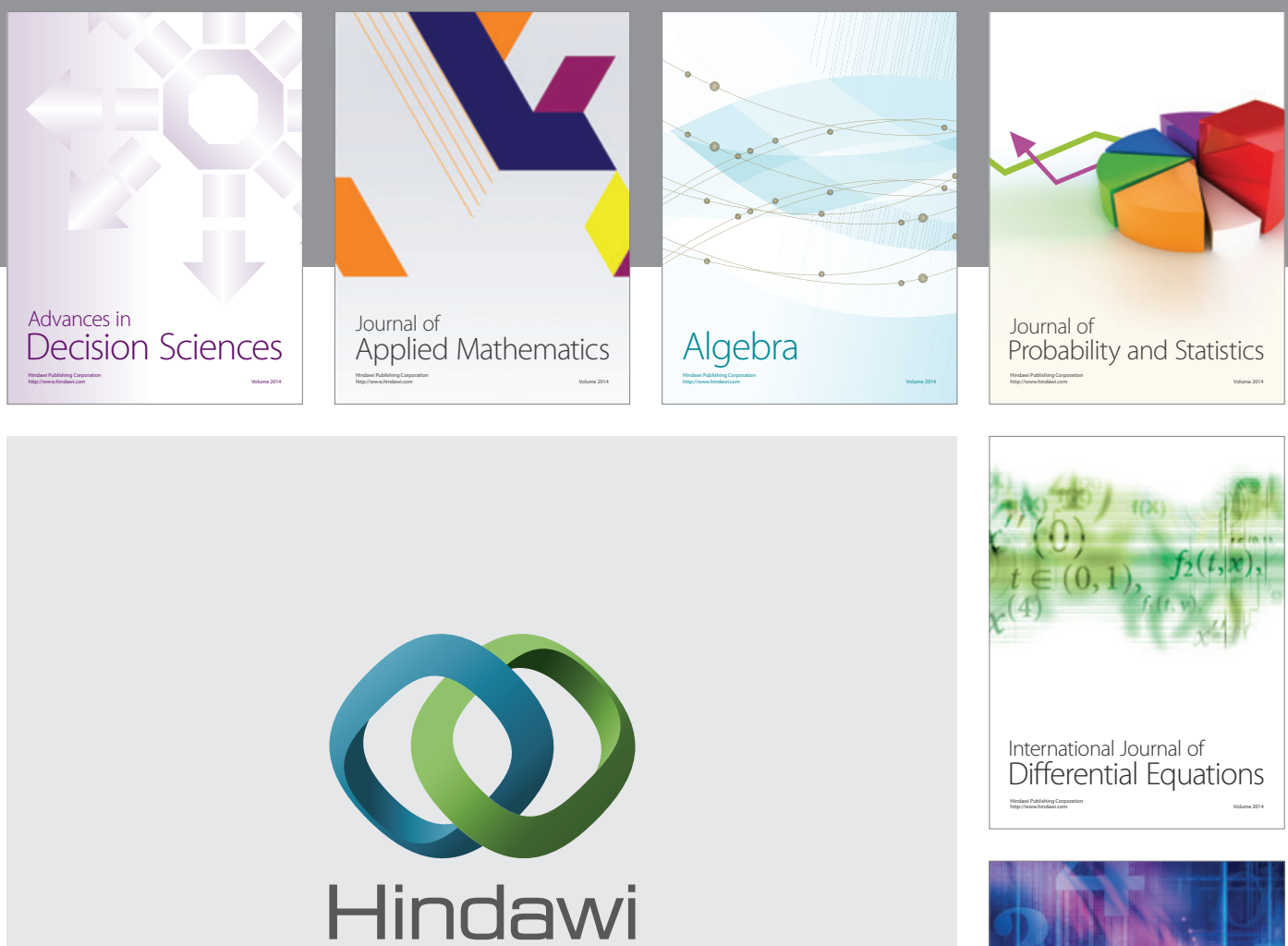

Submit your manuscripts at http://www.hindawi.com
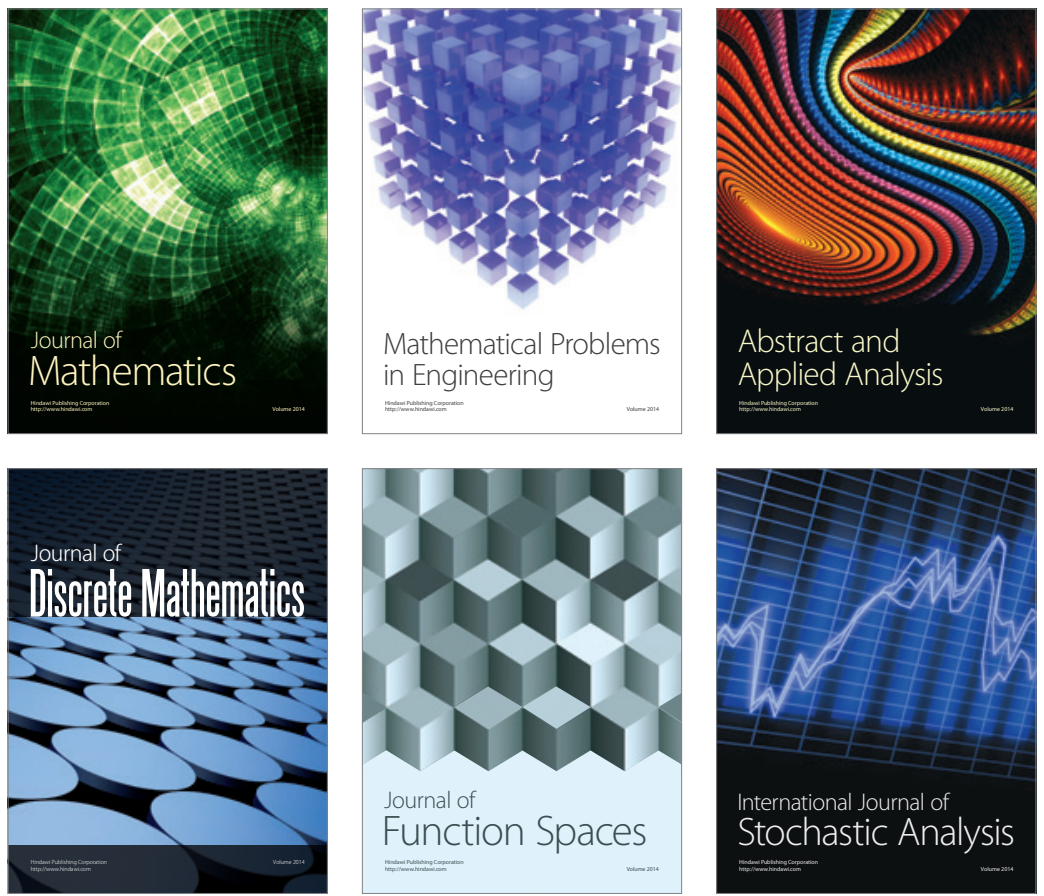

Journal of

Function Spaces

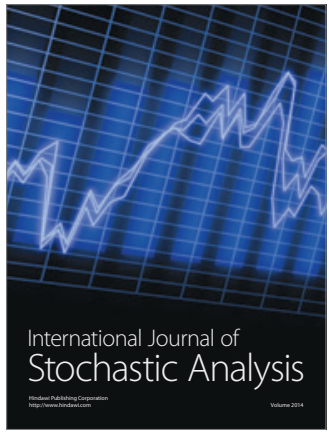

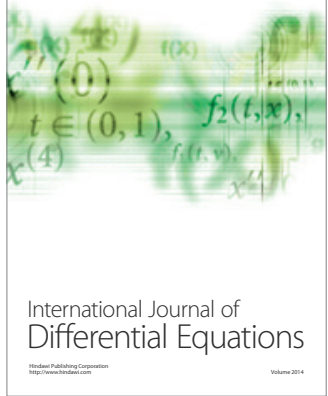
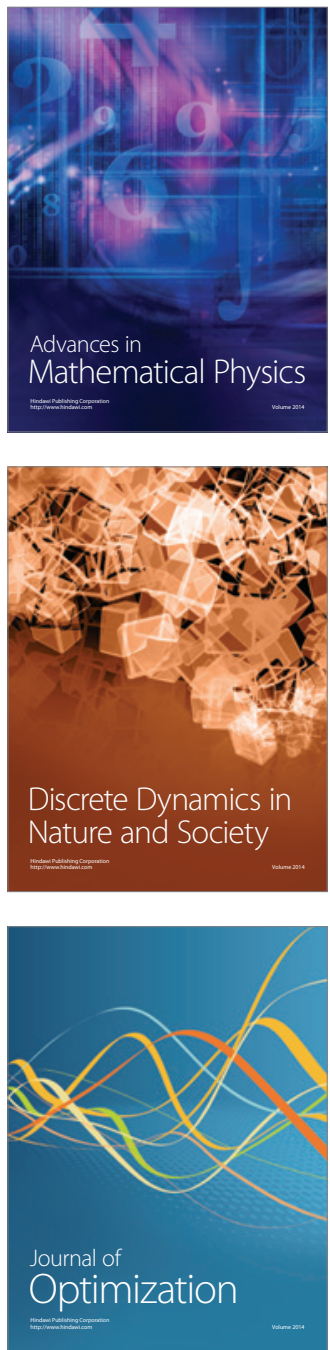